\title{
Ensuring the Operational Health of Droplet-Based Microelectrofluidic Biosensor Systems
}

\author{
Fei Su, Student Member, IEEE, Sule Ozev, Member, IEEE, and Krishnendu Chakrabarty, Senior Member, IEEE
}

\begin{abstract}
Recent events have heightened the need for fast, accurate, and reliable biological/chemical sensor systems for critical locations. As droplet-based microelectrofluidic sensor systems become widespread in these safety-critical biomedical applications, reliability emerges as a critical performance parameter. In order to ensure the operational health of such safety-critical systems, they need to be monitored for defects, not only after manufacturing, but also during in-field operation. In this paper, we present a cost-effective concurrent test methodology for droplet-based microelectrofluidic systems. We present a classification of catastrophic and parametric faults in such systems and show how faults can be detected by electrostatically controlling and tracking droplet motion. We then present a fault simulation approach based on tolerance analysis using Monte-Carlo simulation to characterize the impact of parameter variations on system performance. Finally, we present experimental results on a droplet-based microelectrofluidic system for a real-time polymerase chain reaction application.
\end{abstract}

Index Terms-Microelectrofluidic biosensor systems, reliability, testing for manufacturing and operational defects.

\section{INTRODUCTION}

$\mathbf{R}$ ECENT events have heightened the need for new technologies for combating bio-terrorism threats and for monitoring environmental toxins. In a likely scenario, a dangerous pathogenic microbe, such as the variola virus that causes smallpox, may be spread in a densely-populated area such as an airport. It takes 12 to 14 days for the appearance of the first symptoms of smallpox, making it possible for hundreds or thousands of people to be exposed before the attack is detected. It has been recognized that an undetected bio-attack could easily become a national outbreak [1]-[3]. Therefore, the real-time detection and identification of pathogenic bio-molecular agents in the field is key to an effective homeland security infrastructure. There is a pressing need for fast, accurate, and reliable biological/chemical sensing and detecting systems for critical locations, such as airports or subway stations. Such systems, capable of continuously sampling and testing air or water samples for biological or chemical warfare toxins and other pathogens, can offer an early warning capability to people before anyone exhibits symptoms.

Bio-molecular detection can be carried out either using immunoassay or DNA-based testing. The former is based on

Manuscript received February 11, 2004; revised July 22, 2004. This work was supported in part by the National Science Foundation under Grant EIA0312352. The associate editor coordinating the review of this paper and approving it for publication was Dr. William Hunt.

The authors are with the Department of Electrical and Computer Engineering, Duke University, Durham, NC 27708 USA (e-mail: fs@ee.duke.edu; sule@ee.duke.edu; krish@ee.duke.edu).

Digital Object Identifier 10.1109/JSEN.2005.848127 an antibody-antigen reaction. An antibody is a highly selective ligand, which is produced by mammalian immunological systems in response to the introduction of an antigen. Nucleic acid testing based on DNA hybridization or DNA sequencing has been employed for commercial biochips. It uses polymerase chain reaction (PCR) to amplify suspect samples, thus making it possible to detect even a single organizm.

In recent years, a novel droplet-based microelectrofluidic system has been developed to analyze nanoliter volumes of agents in real time [4]-[6]. These systems reduce the rate of reagent consumption enabling continuous sampling and analysis for on-line, real-time biological/chemical analysis. By scaling down the concentration of the samples, simple sensing techniques can be utilized to replace conventional, costly, and time-consuming practices involving batch analysis, sample pretreatment and frequent calibration. Advances in this technique make droplet-based microelectrofluidic systems a promising platform in the realm of massively parallel DNA analysis and real-time molecular detection and recognition. The feasibility of performing either protein or DNA real-time reaction on this novel sensing and detecting system has been successfully demonstrated [7]-[9]. Furthermore, these droplet-based microelectrofluidic systems offer the exciting possibility to explore a radically different bio-molecular detection approach by decoupling the problem of determining a binary yes/no response from the problem of determining the agent concentration. By placing such droplet-based microelectrofluidic systems at predetermined detection sites, a series of simple bio-molecular tests, based on colorimetric reactions and optical detection, can be performed. For example, the Coomassie Brilliant Blue method can detect the presence of a protein, which could be the outer coat of a virus or some other infectious agents, by monitoring the change of color [10]. This reaction is rapid and the maximum absorbance occurs within 2 to $5 \mathrm{~min}$. This bio-molecular recognition approach not only reduces the detection time drastically, but it also solves the problem of detecting an unknown agent.

As droplet-based microelectrofluidic systems become widespread in safety-critical biomedical applications, system reliability emerges as an essential performance parameter. In order to ensure availability, e.g., as an always-on "bio smoke alarm," these microelectrofluidic systems should not only be tested adequately after manufacturing, but also need to be monitored continuously during field operation. Therefore, there is a pressing need for efficient testing methodologies that are applicable to both manufacturing and operational defects for such systems. In fact, the 2003 International Technology Roadmap for Semiconductors (ITRS) recognizes the need for new test methods 


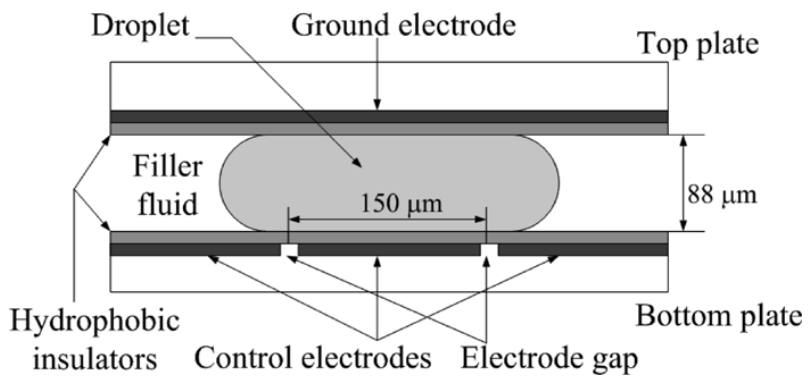

Fig. 1. Actuation mechanism for droplet motion.

for disruptive device technologies that underly microelectromechanical systems (MEMS) and sensors, and highlights it as one of the five most difficult test challenges beyond 2009 [11].

In this paper, we propose a cost-effective test methodology that is applicable both in-field and after manufacturing for droplet-based microelectrofluidic systems. We present an analysis of likely defects in such systems and classify them as catastrophic and parametric faults. We present a novel unified fault detection mechanism for both catastrophic and parametric faults. Fault detection is based on tracking droplet movement through the unused portions of the system. It can be implemented without disrupting the normal mode of operation, and with negligible hardware overhead. Then, we present tolerance analysis based on Monte-Carlo simulation to characterize the impact of variations in physical and fluidic parameters on the system performance. Finally, we present experimental results on a droplet-based microelectrofluidic system for a real-time PCR application, where three distinct physical defects are evaluated in terms of their detection capabilities.

\section{Electrowetting-ActuAted Droplet-BASED MICROELECTROFLUIDIC SYSTEMS}

Electrowetting-based actuation of microelectrofluidic systems has recently been proposed for optical switching [12], chemical analysis [4], and rotating yaw rate sensing [13]. By varying the electrical potential along a linear array of electrodes, electrowetting can be used to move nanoliter volume liquid droplets along this line of electrodes [4]. Droplets can also be transported, in user-defined patterns and under clocked-voltage control, over a two-dimensional array of electrodes without the need for pumps and valves. The basic component of a droplet-based microelectrofluidic system is shown in Fig. 1. The droplet, usually containing biomedical samples, and the filler medium, such as the silicone oil, are sandwiched between two parallel glass plates. The bottom plate contains a patterned array of individually controllable electrodes, while the top plate is coated with a ground electrode. The hydrophobic dielectric insulator is added to the top and bottom plates to decrease the wettability of the surface and to add capacitance between droplet and control electrode.

The basic principle of microdroplet transportation is to electrostatically control the interfacial tension at the droplet/insulator interface. A control voltage is applied to an electrode adjacent to the droplet and at the same time the electrode just under the droplet is deactivated. This causes an accumulation of charge in the droplet/insulator interface, resulting in a surface tension

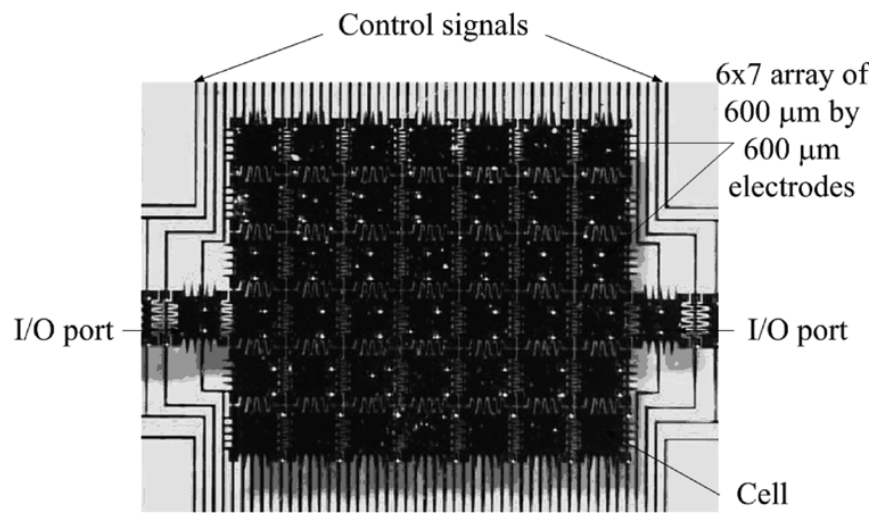

Fig. 2. Two-dimensional electrode array for microfluidics.

gradient across the gap between the adjacent electrodes, which consequently causes the transportation of the droplet. The velocity of the droplet can be controlled by adjusting the control voltage $(0 \sim 90 \mathrm{~V})$, and droplets can be moved at speeds of up to $20 \mathrm{~cm} / \mathrm{s}$. Based on this principle, microfluidic droplets can be moved freely to any location of a two-dimensional array; see Fig. 2 [14]. This design is ideally suited for a large-scale integrated microfluidic system, which is expected to be extensively used for biomedical applications, such as DNA sequencing and bimolecular detection, in the near future. The electrodes can be viewed as building blocks in nanotechnology such that huge networks of nano devices can be easily assembled. Each electrode set can be viewed as a pump at the nano/pico-scale, and an array of these pumps can be used for biochemical assays.

Fluid droplets are introduced to the device from the input-output (I/O) ports on the boundary of an array. Droplets in the array have identical volumes. Hence, this device is called a unit-flow device. It is desirable to maintain the unit-flow constraint since the rate of chemical and biomedical reaction grows exponentially with the growth of droplet volume [4]. In a unit flow environment, the routes that droplets travel and their rendezvous points are programmed into a micro-controller that controls the voltages of the electrodes.

\section{RELATED PRIOR WORK}

Over the past decade, the focus in testing research has broadened from logic and memory test to include the testing of analog and mixed-signal circuits. MEMS is a relatively young field compared to IC design [15], [16], and MEMS testing is still in its infancy. Recently, fault modeling and fault simulation in surface micromachined MEMS has received attention [17], [18]. Researchers in Carnegie Mellon University, Pittsburgh, PA, are developing a comprehensive testing methodology for a class of MEMS known as surface micromachined sensors.

However, test techniques for MEMS cannot be directly applied to microelectrofluidic systems, since the techniques and tools currently in use for MEMS testing do not handle fluids. Hence, they are of limited use for testing microfluidic devices. Most recent work in this area has been limited to the testing of continuous-flow microfluidic systems [19]-[21]. Researchers at the MESA+ Research Institute of the University of Twente, Twente, The Netherlands, have applied mixed-signal testing techniques to the problem of testing a 
microanalysis system. Also, a design-for-testability (DFT) technique for flow-FET-based microfluidic systems has been proposed [21]. Similar to MOSFET, a flow-FET has source and drain electrodes over which a relatively large voltage $(\sim 100$ $\mathrm{V})$ is applied. Due to the principle of electro-osmotic flow, the electric field moves the charge accumulated between the fluid and the surface of channel, dragging the bulk liquid through the channel. This type of microfluidic systems belongs to the category of continuous-flow systems where fluid motion constitutes continuous streams as opposed to discrete droplets. Testing of discrete droplet-based fluidic systems, both off-line and in-field, has not received much attention to date, as these systems have been introduced very recently.

\section{Classification of Faults in Droplet-Based MICROELECTROFLUIDIC SYSTEMS}

It is evident that droplet-based microelectrofluidic systems exhibit behavior resembling analog and mixed-signal devices. Therefore, we classify the faults in these systems as being either catastrophic or parametric, along the line of fault classification for analog circuits [22]. Catastrophic (hard) faults lead to a complete malfunction of the system, while parametric (soft) faults cause a deviation in the system performance. A parametric fault is detectable only if this deviation exceeds the tolerance in system performance. Due to their underlying mixed technology and multiple energy domains, microelectrofluidic systems exhibit failure mechanisms and defects that are significantly different from the failure modes in analog integrated circuits.

Catastrophic faults in microelectrofluidic systems may be caused by the following physical defects.

- Dielectric breakdown: The breakdown of the dielectric at high voltage levels creates a short between the droplet and the electrode. As a result, no charge can be stored in the interface. As the electrowetting mechanism depends on the amount of energy stored in the capacitor formed by the electrode and the droplet, dielectric breakdown inhibits fluid motion.

- Short between the adjacent electrodes: As a result of a short circuit between two adjacent electrodes, these electrodes effectively form one longer electrode. Thus, the droplet residing on this electrode is no longer large enough to overlap with the adjacent electrodes, inhibiting its actuation.

- Degradation of the electrode: This degradation effect is unpredictable and may become catastrophic during the operation of the system. Fig. 3 illustrates electrode degradation due to the insulator degradation defect [14]. A consequence of electrode degradation is that droplets often fragment and their motion is prevented because of the unwanted variation of surface tension forces along their flow path.

- Open in the metal connection between the electrode and the control source: This defect results in a failure of charging electrode while trying to drive the droplet.

Physical defects that cause parametric faults include the following.

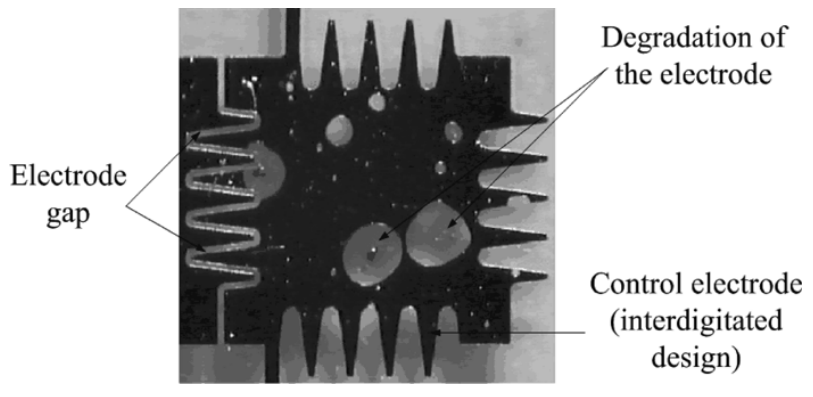

Fig. 3. Top-level view of a cell: Electrode degradation.

- Geometrical parameter deviation: The deviation in insulator thickness, electrode length, and height between parallel plates may exceed their tolerance value.

- Insulator degradation: This "wear-and-tear" defect may become apparent gradually during operation. If left undetected, it may eventually cause electrode degradation.

- Particle contamination: During in-field operation of a microelectrofluidic system, the droplet or the filer fluid may be contaminated by a particle, such as a dust particle or a foreign fluid droplet. Typically, such particles are then attached to the surface of the insulator of a cell and affect the motion of the droplet.

- Change in viscosity of droplet and filler medium. These deviations can occur during the operation due to an unexpected biochemical reaction, or a defect in the control system causing unwanted temperature variation.

Faults in microelectrofluidic systems can also be classified based on the time at which they appear. Therefore, system failure or degraded performance can either be caused by manufacturing defects or by parametric variations. Testing of manufacturing defects, such as a short between the adjacent electrodes or a deviation in the value of the geometrical parameters, should be performed immediately after production. However, operational faults, such as degradation of the insulator or change in fluid viscosity, can occur throughout the lifetime of the system. Therefore, concurrent testing during system operation is essential to ensure the operational health of safety-critical systems.

\section{UNIFIED DETECTION MeChanism}

In the proposed testing methodology, test droplets (e.g., 0.1-M KCL) are released into the microelectrofluidic system from droplet sources and are guided through the system following the designed testing scheme. Both catastrophic and parametric faults are detected by electrostatically controlling and tracking the motion of these test stimuli droplets. This testing method is minimally invasive and easy to implement, which offers an opportunity to eradicate the need for expensive and bulky external devices.

To facilitate a decision-making process, a detection mechanism is needed for both catastrophic and parametric faults. This mechanism needs to be based on a pass/fail criterion that yields the some response to each of the possible faults to prevent masking among various types of faults. The proposed unified detection mechanism consists of a simple RC oscillator circuit 


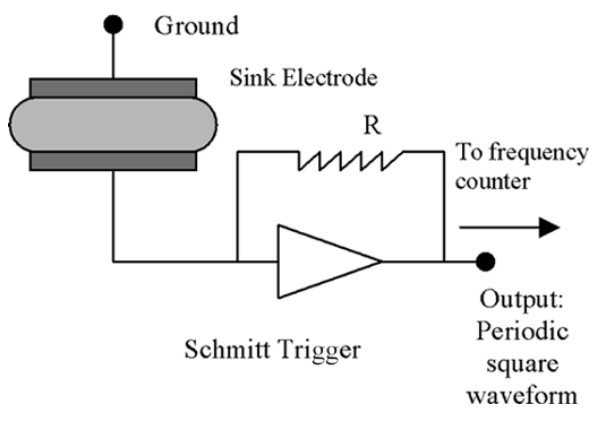

Fig. 4. Simple R-C oscillator circuit.

formed by the sink electrodes and the fluid between them as an insulator; see Fig. 4. The capacitance of this structure depends on the presence of the droplet since the filler medium and the droplet have distinct permittivities. By sensing the capacitance of this structure through a simple frequency counter, one can determine whether a droplet has reached the sink. This mechanism can be electronically implemented and easily integrated on chip. In order to provide a unidirectional and unambiguous detection mechanism, the pass/fail criterion has to be determined based on the presence of the droplet at the sink electrode and this criterion should be applied for all test cases. In this paper, we associate the fault-free operation with the presence of the droplet at the sink electrode and faulty operation with its absence.

\section{A. Online Testing of Catastrophic Faults}

Most catastrophic faults cause a complete cessation of droplet transportation at the system level. Therefore, we can easily detect these faults by using the testing scheme outlined in Fig. 5. The fault site in this two-dimensional array is highlighted. Droplets are first driven along one direction, e.g., along the $x$ axis, and they are observed at the other end of the array. Each row of the array transports a single droplet of fluid. Due to the catastrophic fault in Row 3, no droplet is observed for this row. As a result, the cells in this row are deemed as candidate faulty cells. Next, droplets are driven along the $y$ axis, and due to the fault in the array, no droplet is observed at the other end of Column 3. Thus, we conclude that Column 3 contains a faulty cell. From the information about the faulty row and column, we can uniquely identify the faulty cell in the array.

The above illustration assumes that a catastrophic fault affects only one cell of the array. The testing technique described here can, however, be extended for locating multiple faulty cells, e.g., through the use of multistep adaptive fault location methods. An important advantage of this approach is that it can be integrated into the droplet-manipulation-based microfluidic steps underlying a biomedical reaction, e.g., polymer chain reaction [23]. Concurrent testing can be carried out simultaneously with a biochemical reaction utilizing unused cells in the array, and a degree of fault tolerance can be achieved by reconfiguring the array such that the droplets avoid faulty cells in their flow paths.

\section{PARAmetric Fault Testing}

While catastrophic faults have the highest priority for detection as they result in complete malfunction, parametric faults are much harder to detect and may result in malfunction depending

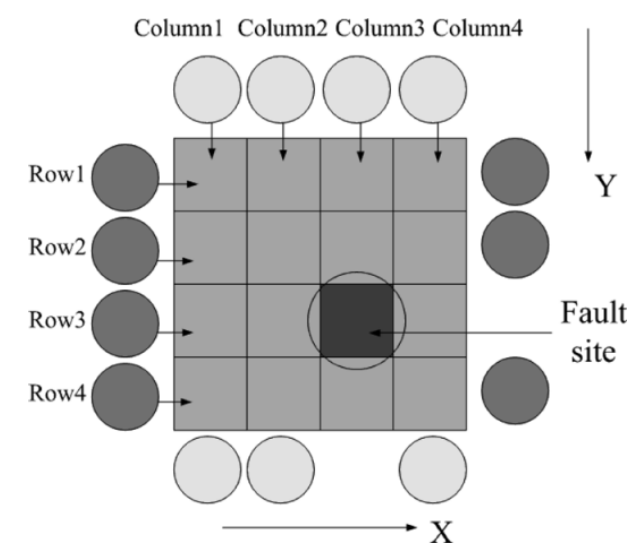

Fig. 5. Illustration of the catastrophic fault testing scheme.

on the application domain and specifications. As a result, a viable concurrent testing scheme needs to consider both catastrophic and parametric faults. A large set of parametric faults that can arise from environmental effects, or from the degradation of system materials, may eventually lead to system failure.

The degradation of the insulator thickness and changes in the viscosity of the fluids are examples of such parametric faults that do not necessarily result in imminent loss of droplet movement, but rather result in degraded performance. For example, an increase in the viscosity of the filler fluid might impede droplet motion, resulting in undesired fluid concentrations at the mixers. The identification of such deviations is essential during in-field operation in droplet-based microelectrofluidic systems. The analysis of fault manifestations requires an understanding of the fault-free behavior and the variations in this behavior with respect to the variations in underlying parameters.

\section{A. Fault-Free Model}

The behavioral model of droplet-based microelectrofluidic systems is based on the electrowetting actuation principle. We assume that the liquid flow within the droplet can be approximated as laminar flow, which requires that the Reynolds number of the droplet fluid be less than 1000 . This assumption is valid because the Reynolds number of a nanoliter of fluid is usually no larger than 100. The principle underlying this model is to balance the work done by the surface tension gradient force with the power dissipation. The power dissipation during the droplet transportation is caused by three factors: the viscosity and the resistance of the filler medium, the friction around the droplet/insulator surface and the internal viscous flow of the droplet. The following analytical model for droplet motion is derived in [24]

$\frac{\varepsilon_{0} \varepsilon_{R}}{2 d} V^{2}-F_{T}=B\left(\frac{\mu_{d} U}{\gamma_{L M}}\right)^{0.3} \gamma_{L M}+\left(\frac{\mathrm{mL}}{h}+s\right) \mu_{0} U+\zeta U$

where $V$ is the control voltage, $U$ is the transport velocity, $\varepsilon_{0}$ is permittivity of free-space, $\gamma_{L M}$ is the liquid-medium interfacial tension constant, and the remaining parameters and coefficients are as described in Tables I and II. Equation (1), covering all the significant physical phenomena in electrowetting, shows that the transport velocity of the droplet is a function of the control voltage and a number of relevant physical and fluidic 
TABLE I

Physical Parameters AFFECTING THE DROPLET Velocity

\begin{tabular}{ll|l|l}
\hline $\begin{array}{l}\text { Parameter } \\
\text { name }\end{array}$ & Parameter description & $\begin{array}{l}\text { Nominal } \\
\text { value }\end{array}$ \\
\hline $\begin{array}{ll}d & (\mu \mathrm{m})\end{array}$ & Thickness of dielectric layer & 1 \\
\hline$L$ & $(\mu \mathrm{m})$ & Electrode length & 150 \\
\hline$h \quad(\mu \mathrm{m})$ & $\begin{array}{l}\text { Height between two parallel } \\
\text { plates }\end{array}$ & 88 \\
\hline$\mu_{0} \quad(\mathrm{cP})$ & Viscosity of filler fluid & 1.7 \\
\hline$\mu_{d}$ & $(\mathrm{cP})$ & Viscosity of droplet & 1.9 \\
\hline
\end{tabular}

TABLE II

NuMERICAL COEFFICIENTS AFFECTING THE DROPLET VELOCITY

\begin{tabular}{l|l|l}
\hline Coefficient name & $\begin{array}{l}\text { Coefficient } \\
\text { description }\end{array}$ & Value \\
\hline$F_{T} \quad($ dyne $/ \mathrm{cm})$ & Threshold & 2.47 \\
\hline$B$ & Droplet viscous effect & 0.55 \\
\hline$m, s$ & Oil viscous effect & 28,112 \\
\hline$\zeta \quad\left(\right.$ dyne $\left.\mathrm{s} / \mathrm{cm}^{2}\right)$ & Contact-line friction & 0.4 \\
\hline$\varepsilon_{R}$ & $\begin{array}{l}\text { Relative permittivity of } \\
\text { insulator }\end{array}$ & 1.93 \\
\hline
\end{tabular}

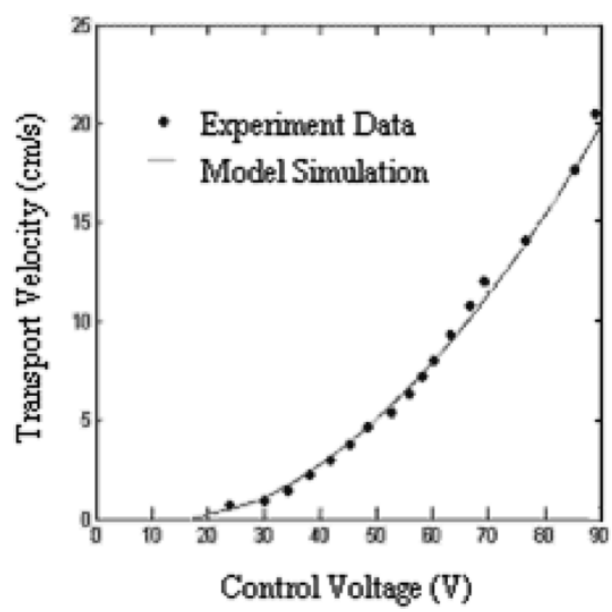

Fig. 6. Variation of transport velocity with control voltage.

parameters. We experimentally verified using laboratory apparatus that the analytical model accurately describes physical behavior. Fig. 6 shows that the experimental data obtained from video frame counting matches simulation data for a wide range of values for the control voltage.

This behavioral model implies that the velocity of the droplet is a function of the environmental parameters, provided that the voltage is kept at a constant value. Thus, a defect involving these parameters results in a deviation of the droplet velocity from the nominal value predicted by (1); see Fig. 7 .

Ideally, at a certain applied voltage, the droplet velocity is precisely determined by (1). However, in practice, all the parameters involved in (1) vary with a certain tolerance, resulting in a range of acceptable values for the droplet velocity, as illustrated in Fig. 8. A viable parametric testing scheme has to take these variations into account to minimize the likelihood of a false alarm. Such parametric variations dictate certain upper and lower bounds on the droplet velocity in a fault-free system. These bounds can be computed through tolerance analysis [25].

Since parametric faults manifest as deviations in droplet velocity, parametric testing mainly consists of ensuring that the droplet velocity is within its bounds. Direct measurement of droplet velocity requires experimental methods such as video frame counting and capacitive sensing. All these methods, however, require external devices such as CCD cameras or oscillator circuits for capacitance measurement, which add unacceptable overhead and make testing cumbersome, especially in concurrent scenarios.

Fortunately, we can determine whether the droplet velocity is within the predetermined bounds through a nonintrusive and cost-effective test setup, which is based on the readouts of droplet ports, and on adjustments in the duration of each electrode pulse.

\section{B. Lower-Bound Testing}

A parametric fault, such as an increase in the viscosity of the filler fluid, may slow the droplet beyond the acceptable value $U_{\min }$. In order to detect such effects, the droplet can be moved between two ports, a source and a sink, such that it can reach the sink only if its velocity is higher than the required minimum. Consider the droplet movement shown in Fig. 9. For the droplet to move from the source to the sink, each electrode actuation has to be in the form of a pulse and the duration of the pulse has to be long enough for the droplet to reach the boundaries of the adjacent electrode. This is the boundary condition (corresponding to $U=U_{\min }$ ) under which the droplet can move continuously. If the actual velocity is larger than $U_{\min }$, the droplet will rapidly traverse through the adjacent electrodes, and wait there until the next switching of control signal. On the other hand, if the velocity is less than $U_{\min }$, the droplet cannot keep up with the switching rate of the control electrodes. This implies that when the droplet reaches one electrode, the adjacent electrode has already been deactivated. For example, in Fig. 9 , if the voltage $V_{3}$ becomes zero before the droplet reaches $X_{2}$, the droplet will be stuck at some point between $X_{1}$ and $X_{2}$.

As a result, the shortest pulse duration $T$ that allows the droplet with the minimum acceptable velocity, $U_{\min }$, to reach its destination is given by: $T=L / U_{\min }$, where $L$ is the electrode length. If the pulse duration is chosen appropriately, the droplets with velocities higher than $U_{\min }$ will reach the sink, and the droplets with lower velocities will fail to complete the movement, as illustrated in Fig. 9.

\section{Upper-Bound Testing}

Upper-bound testing differs from lower-bound testing in the sense that the fault-free case is represented by the failure of a droplet to catch-up with the pulse frequency. However, testing for the upper-bound is more complicated than simply checking whether the droplet fails to reach the target since it violates the unified pass/fail criterion. Moreover, a catastrophic fault that impedes the droplet motion may be perceived as a fault-free operation in this case.

Fortunately, by slightly modifying the lower-bound testing scheme, we can test for the upper-bound of the droplet velocity, 

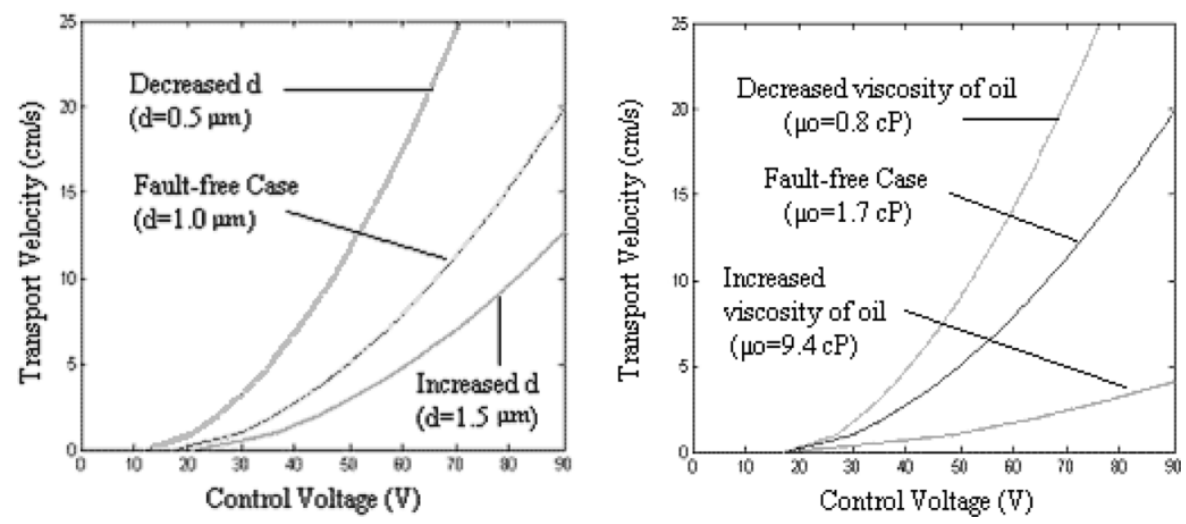

Fig. 7. Effect of parametric variations on droplet velocity.

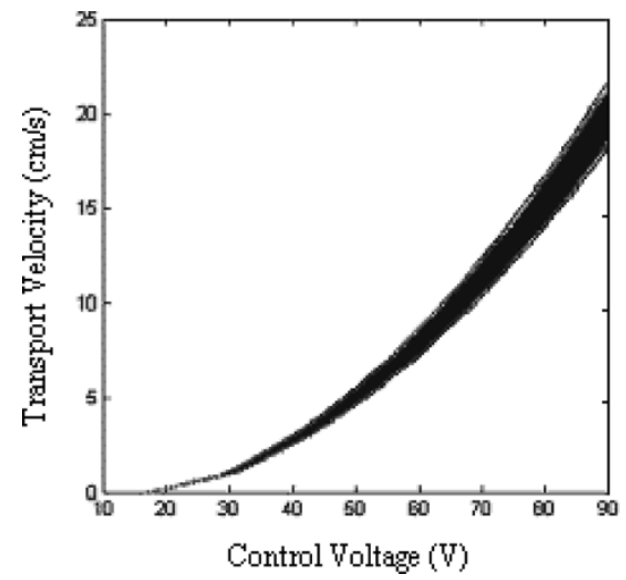

Fig. 8. Range of acceptable values for droplet velocity.

as illustrated in Fig. 10. In this case, bidirectional droplet movement is utilized, where the source and the sink are the same port. In the first phase of the test, pulses of duration $T=L / U_{\max }$ are utilized, where $U_{\max }$ is the precomputed upper bound on the droplet velocity. A droplet with an acceptable velocity $(U<$ $\left.U_{\max }\right)$ will not be able to reach the final electrode; it will be stuck at an intermediate position. In the second phase of the test, which begins right after the final electrode is deactivated, the droplet motion is set in the reverse direction. However, one electrode is skipped so as to keep the droplets that had reached the final electrode from moving. In this phase, the pulse duration is much higher to allow for the slower droplets to continue their motion. This backward pulsation will pick up the droplets that may have been stuck at intermediate points and move them toward the sink. Hence, the detection of a droplet at the end of the operation indicates a droplet velocity that is lower than the allowed maximum, i.e., a fault-free operation.

It is evident that this testing scheme can be combined with catastrophic fault testing to form a unified complete test methodology. It only needs to detect the droplet ports without additional testing devices; this feature dramatically reduces the test cost. In addition, its simplicity facilitates concurrent testing.

\section{Evaluation of the Parametric Test Strategy}

The parametric testing strategy described here relies on the fact that parametric deviations result in an unexpected devia-

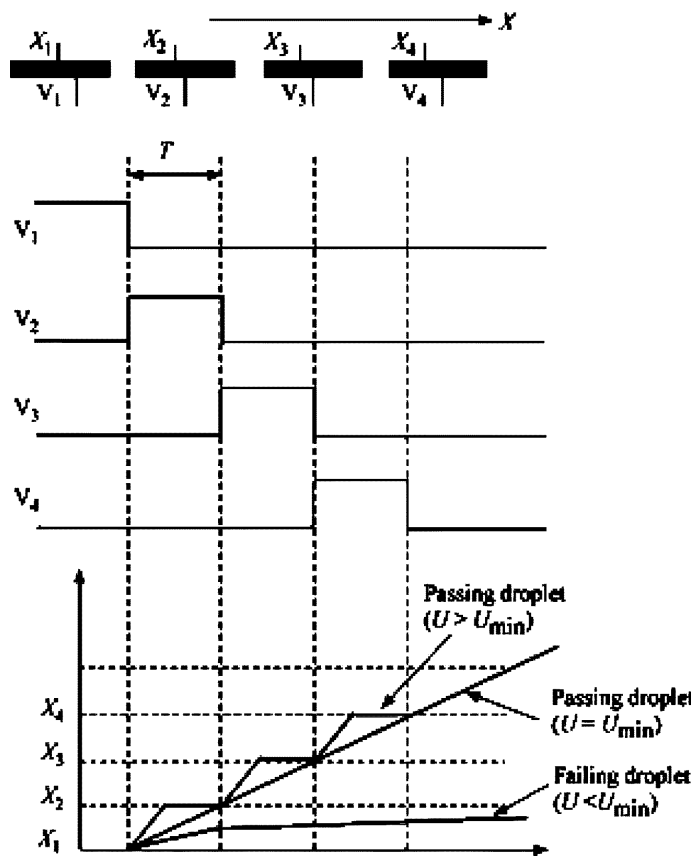

Fig. 9. Source-to-sink droplet motion for lower-bound testing.

Port $1 \longrightarrow$ Phase I $\longrightarrow$ Port 2

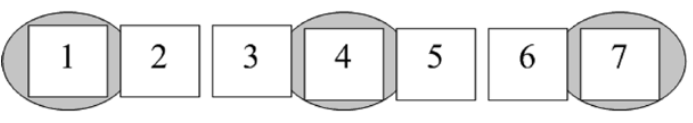

Phase II

Upper bound testing $\begin{gathered}1 \rightarrow 2 \rightarrow \\ \text { Phase I }\end{gathered} \begin{gathered}\rightarrow \rightarrow 4 \rightarrow 5 \rightarrow 6 \rightarrow 7 \rightarrow 4 \rightarrow 3 \rightarrow 2 \rightarrow 1 \\ \text { Phase II }\end{gathered}$

Fig. 10. Two-phase upper testing using bi-directional droplet motion.

tion in the droplet velocity. Equation (1) guarantees that a highenough deviation in each parameter will eventually result in the velocity bound being violated. Hence, the minimum-detectable deviations for all the parameters serve as a good evaluation metric in understanding the viability of the proposed testing scheme.

For a certain amount of deviation in a parameter to be detectable, it needs to shift the droplet velocity outside its determined bounds, while other parameters are allowed to vary 


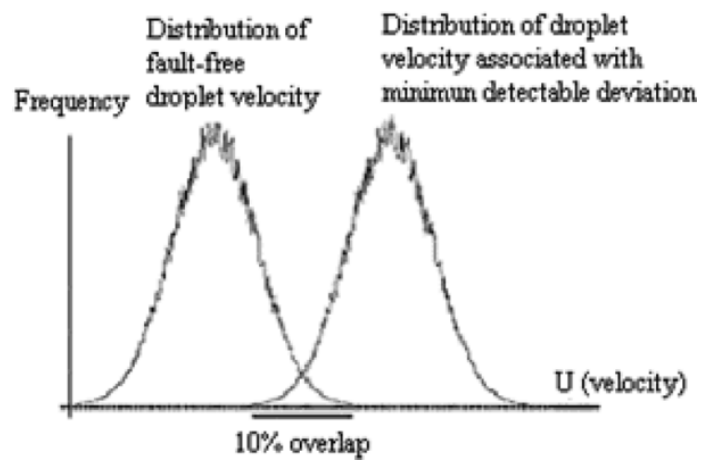

Fig. 11. Detectable parameter deviations must result in a $10 \%$ or less overlap in velocity distributions.

within their tolerance. Since the droplet velocity for both the fault-free and faulty systems can be expressed in the terms of ranges, the detection of a certain parametric deviation can statistically be assured if droplet velocity ranges for the faulty and fault-free systems are nonoverlapping. However, since most physical parameters exhibit a Gaussian-like distribution, the nonoverlapping criterion can be relaxed to include a $10 \%$ overlap, as illustrated in Fig. 11, leading to a detection probability higher than $99.9 \%$.

\section{E. Evaluation of Detectability}

In order to illustrate the viability of the parametric testing scheme, we have conducted simulation-based experiments and computed the velocity tolerance, as well as minimum-detectable deviation values for each physical parameter.

1) Tolerance Analysis: It is noted that the equation governing the relation between transport the velocity and the control voltage is nonlinear. The simplest and the most popular method for nonlinear statistical tolerance analysis is the Monte-Carlo simulation method [25]. Random values for each parameter are generated according to its distributions, and the value of the response function is computed for each set of parameter values. By generating very large samples, the tolerance response can be statistically analyzed.

In our case, it is assumed that all parameters, including geometrical parameters $(d, L, h)$ and fluidic parameters $\left(\mu_{0}\right.$, $\mu_{d}$ ), follow a Gaussian distribution with a mean value $\mu$ and a standard deviation $\sigma$. Using a $5 \%$ tolerance for each physical and fluidic parameter, Monte-Carlo analysis has been carried out through MATLAB simulations (1000 runs). Fig. 12 shows the results of the Monte-Carlo analysis over a range of control voltage values. For high control voltage values, the deviation in droplet velocity is higher as the sensitivity of parameter $d$ increases with the square of the control voltage, making this parameter the dominant variable. For a nominal control voltage value of $50 \mathrm{~V}$, the transport velocity tolerance is determined to be $7 \%$.

2) Minimum Detectable Deviations: In order to compute the minimum detectable deviations (MDDs) for each parameter, a linear search algorithm in conjunction with Monte-Carlo simulations is utilized, as illustrated in Fig. 13. Even though linear search is quite inefficient, computational complexity is not an issue here due to the small number of parameters.

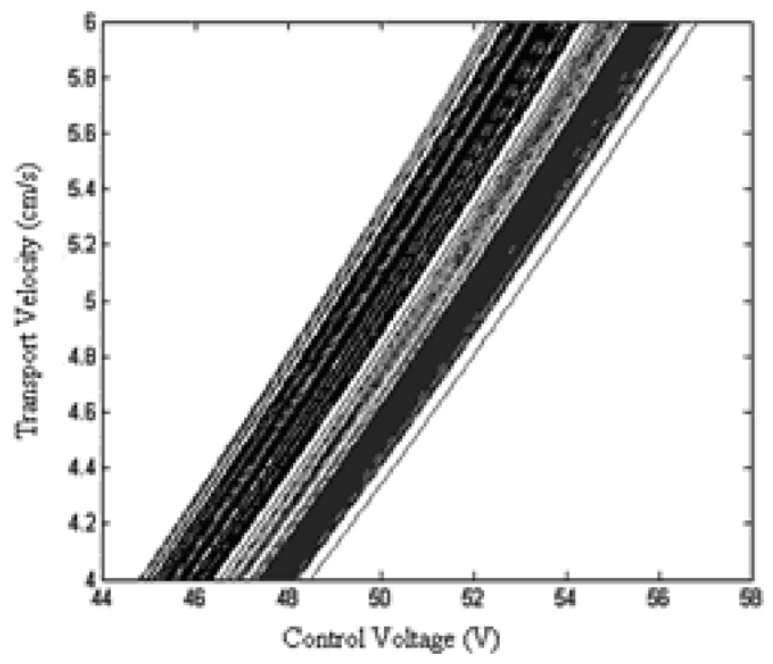

Fig. 12. Results obtained with Monte-Carlo simulations.

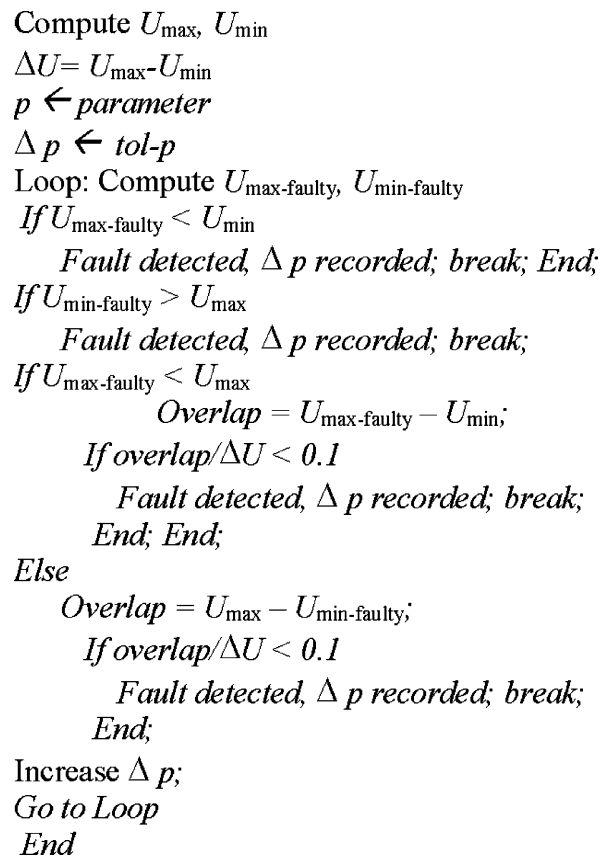

Fig. 13. Linear search algorithm for computing the MDD of a parameter.

In order to determine the MDD for a parameter, $p$, we start with a deviation $\Delta p$ that exceeds the tolerance of $p$. Through Monte-Carlo simulations, the upper and lower bounds on the resulting transport velocity are determined. If the overlap between the fault-free and faulty ranges of the transport velocity is less than $10 \%$, the corresponding deviation $\Delta p$ is considered to be detected. Since the actual distributions are Gaussian like, such an overlap results in less than $0.1 \%$ probability of misclassification in terms of both false positives and false negatives.

Table III shows the MDD values for the physical and fluidic parameters for a control voltage of $50 \mathrm{~V}$. For this particular setup, the thickness of the dielectric layer $(d)$ has the most impact on the transport velocity. Thus, even small variations in $d$ can be detected using the proposed concurrent detection method. On the other extreme, the viscosity of the droplet $\mu_{d}$ has the least impact on the transport velocity, leading to a high MDD value. It is worthwhile to note that the viscosity of the 
TABLE III

MiNIMUM DETECTABLE DEVIATIONS

\begin{tabular}{ll|l|l}
\hline \multicolumn{2}{l|}{ Parameter } & Nominal & MDD \\
\hline$d \quad(\mu \mathrm{m})$ & 1 & $8.5 \times 10^{-2}(8.5 \%)$ \\
\hline$L$ & $(\mu \mathrm{m})$ & 150 & $91(60.7 \%)$ \\
\hline$h$ & $(\mu \mathrm{m})$ & 88 & $33(37.5 \%)$ \\
\hline$\mu_{0}(\mathrm{cP})$ & 1.7 & $0.28(16.5 \%)$ \\
\hline$\mu_{d}(\mathrm{cP})$ & 1.9 & $11(5.8 \times 100 \%)$ \\
\hline
\end{tabular}

filler medium $\mu_{0}$ and the viscosity of the droplet $\mu_{d}$ are closely related, and are affected by the same environmental changes. As a result, a change in $\mu_{d}$ is associated with a similar change in $\mu_{0}$ and, thus, is detected at a much lower deviation than as suggested in Table III. In addition, by using various control voltage values, the sensitivities of a number of parameters can be adjusted. This multiphase testing scheme deceases the MDD values for all parameters involved.

\section{EXPERIMENTAL SET-UP}

In this section, we apply the previous analysis of detectability evaluation to test multiple parametric faults and present the experimental results for a droplet-based microelectrofluidic system for real-time PCR applications.

\section{A. Real-Time PCR in Droplet-Based Microelectrofluidic Systems}

The polymerase chain reaction (PCR) is used to amplify the copies of specific fragments of DNA and is a key technique for DNA-based bio-molecular detection [26]. There are three major steps in PCR, which are repeated for 30 to 40 cycles. First, the target genetic material must be denatured, i.e., the double strands of its helix must be unwound and separated into single stranded DNA by heating it to $94^{\circ} \mathrm{C}$. The second step is hybridization or annealing, in which the primers bind to their complementary bases on the now single-stranded DNA at $54{ }^{\circ} \mathrm{C}$. The third is DNA synthesis by a polymerase. $72{ }^{\circ} \mathrm{C}$ is the ideal working temperature for the polymerase. Starting at the annealed primer, the polymerase can read a template strand and match it with complementary nucleotides very quickly. This step generates two new helixes, each of which is composed of one of the original strands plus its newly assembled complementary strand. Because both strands are copied during PCR, there is an exponential increase of the number of copies of the genes. An experimental investigation has been conducted to determine the suitability of droplet-based microelectrofluidic systems for microfluidic PCR application [8]. Within such electrowetting-actuated systems, real-time PCR assays in 300 $\mathrm{nL}$ droplets have been successfully performed. Techniques for modeling and optimization of PCR at the system level have been also described in the literature [23], [27].

\section{B. Testing Parametric Faults in PCR Microelectrofluidic Systems}

In order to increase the reliability of the real-time PCR application, which is critical to DNA-based bio-molecular detection, the possible faults in such microelectrofluidic systems need to be tested not only after manufacturing, but also during in-field operation. Parametric faults, arising from degradation of system materials or environmental effects, are much harder to detect than catastrophic faults. Here we analyze the testing for some parametric faults in such systems in terms of their MDD values. The nominal values of system parameters are given in Table I, except for viscosities of the droplet and the filler fluid $\left(\mu_{0}\right.$ and $\mu_{d}$ ), which change due to the different application temperatures of PCR.

1) Insulator Degradation: As described in Section III, this "wear-and-tear" defect may appear gradually during the in-field operation of a microelectrofluidic system. We can consider this degradation as a global defect and model its effect in the parameters $d$ and $h$. Thus, the degradation of the insulator (denoted as $\Delta d$ ) causes two inversely-corrected geometrical parametric faults, i.e., decreasing the insulator thickness $(d)$ and increasing the height between parallel plates $(h)$. In order to break the dependency between $d$ and $h$, we introduce an independent manufacturing variable $H$ to denote the overall height of the two-electrode structure. With this convention, $d$ and $h$ are the independent variables during manufacturing and $h=H-d$. However, after manufacturing, any degradation of the insulator affects both $d$ and $h$; thus, the dependency needs to be incorporated into the random sampling process for Monte-Carlo simulations. In order to find the minimum value of $\Delta d$ that causes a fault alarm, the Monte-Carlo sampling needs to be modified as follows.

1) Sample $d$ and $H$ independently, then calculate $h$ as $h_{\text {calculated }}=H_{\text {sampled }}-d_{\text {sampled }}$.

2) Insert the degradation defect $\Delta d$ into $d: d=d_{\text {sampled }}-$ $\Delta d$; set $h=h_{\text {calculated }}+\Delta d$.

3) Sample all other variables according to their rules (same with Section 5.5).

4) The linear search algorithm in Fig. 12 is applied to find the minimum value of $\Delta d$ causing an alarm, and record it.

Monte-Carlo simulations indicate that the minimum detectable degradation of insulator is $0.051 \mu \mathrm{m}$ (i.e., $\Delta d / d=5.1 \%$ ). Therefore, even a small degradation during the operation is easily detectable. This approach is also applicable to the analysis of degraded performance caused by the deviation in insulator thickness during the manufacturing process.

2) Particle Contamination: During in-field operation of PCR in a microelectrofluidic system, the droplet or the filler fluid may be contaminated by a particle, such as a dust particle or a foreign fluid droplet. Typically, such particles are then attached to the surface of the insulator of a cell since they are not large enough to move with electrowetting. As a dust particle is likely to be smaller than droplet in size, this defect should be modeled as a point defect, not a global defect; see Fig. 14(a). Similar to the insulator degradation case, this defect causes the deviation of two geometrical parameters $d$ and $h$. However, the analysis of these two parametric faults is more complicated than a global insulator degradation. Equation (1) of the fault-free model indicates that the surface tension gradient force is proportional to the electrostatic energy $\left((1 / 2) C V^{2}\right)$, which is stored in the capacitor formed between the conductive 


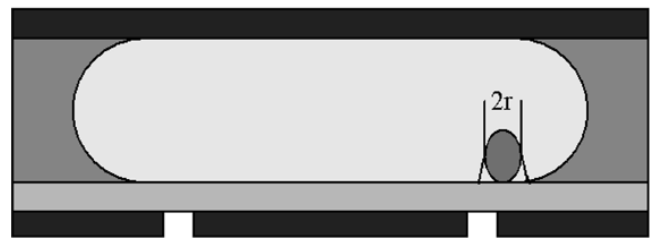

(a)

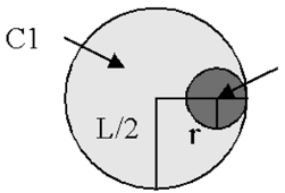

(b)

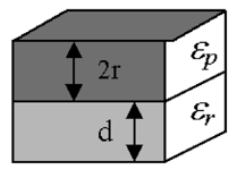

(c)

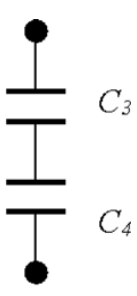

Fig. 14. Particle contamination on the insulator surface. (a) Side view. (b) Top view: $C_{\text {eff }}=C_{1}+C_{2}$. (c) Equivalent capacitor model: $1 / C_{2}=1 / C_{3}+1 / C_{4}$.

droplet and the control electrode when a voltage $V$ is applied. The main contribution to $C$ is from the insulator layer, i.e., $C=\left(\varepsilon_{r} \varepsilon_{0} / d\right) A$, where $\varepsilon_{r}$ is the relative permittivity of the insulator, and $A$ is the area of the interface between the droplet and the insulator and approximated to be $\pi(L / 2)^{2}$. Here, the effect of the dust particle on the capacitance $C$ can be modeled as $C_{\mathrm{eff}}=C_{1}+C_{2}$, where $C_{2}$ is the capacitance of the insulator area covered by the particle and $C_{1}$ is the capacitance of the remaining structure; see Fig. 14(b). We then use an equivalent capacitor model to obtain $C_{2}$, which is considered as a series of two capacitors $C_{3}$ and $C_{4}$, i.e., $1 / \mathrm{C}_{2}=1 / C_{3}+1 / C_{4}=$ $\left(2 r / \varepsilon_{P} \varepsilon_{0} A^{\prime}\right)+\left(d / \varepsilon_{r} \varepsilon_{0} A^{\prime}\right)=\left(1 / \varepsilon_{0} A^{\prime}\right)\left(\left(2 r \varepsilon_{r}+d \varepsilon_{P}\right) / \varepsilon_{r} \varepsilon_{P}\right)$, where $r$ is the radius of the particle, $\varepsilon_{p}$ is the relative permittivity of the particle, and $A^{\prime}$ is the area of the interface between the particle and the insulator and approximated to be $\pi r^{2}$; see Fig. 14(c). Therefore

$C_{\mathrm{eff}}=C_{1}+C_{2}=\frac{\varepsilon_{r} \varepsilon_{0}}{d}\left(\pi\left(\frac{L^{2}}{4}\right)-\pi r^{2}\right)+\frac{\varepsilon_{r} \varepsilon_{r} \varepsilon_{P}}{2 r \varepsilon_{r}+d \varepsilon_{P}}\left(\pi r^{2}\right)$.

We would like to reflect the charge in the effective capacitive onto one of the variables involved in the fault-free model $d$. Therefore

$$
\begin{aligned}
C_{\mathrm{eff}} & =\frac{\varepsilon_{r} \varepsilon_{0}}{d_{\mathrm{eff}}}\left(\pi\left(\frac{L^{2}}{4}\right)\right) \text { and } \\
d_{\mathrm{eff}} & =\frac{\varepsilon_{r}\left(\frac{L^{2}}{4}\right)}{\frac{\varepsilon_{r}}{d}\left(\frac{L^{2}}{4}-r^{2}\right)+\frac{\varepsilon_{r} \varepsilon_{p}}{d \varepsilon_{p}+2 r \varepsilon_{r}}\left(r^{2}\right)} .
\end{aligned}
$$

In order to analyze the minimum detectable size $(r)$ of this dust particle, Monte-Carlo simulation needs to be modified by taking into account its impact on the overall insulator properties. Thus, the Monte-Carlo sampling procedure needs to be modified as follows.

1) $d, L$, and $H$ are sampled independently, then calculate $h$ as $h_{\text {calculated }}=H_{\text {sampled }}-d_{\text {sampled }}$.

2) Using (2), calculate $d_{\text {eff }}$; set $\Delta d=d_{\text {eff }}-d_{\text {sampled }}$.

3) Calculate the effective height $h_{\mathrm{eff}}$ by $h_{\mathrm{eff}}=h_{\text {calculated }}-$ $\Delta d$.

4) Sample all other variables according to their rules (same with Section 5.5).

5) Apply the linear search algorithm to find the minimum value of $r$ causing an alarm.

The minimum detectable radius of particle is found to be $22 \mu \mathrm{m}$ when $\varepsilon_{p}$ is set to be 10 . Thus, with this methodology, contaminates of size larger than $2 \%$ of the droplet size can be detected. The manufacturing defect that causes a bulge in insulator surface can also be analyzed by this method with setting $\varepsilon_{p}$ to be $\varepsilon_{r}$. In this case the minimum detectable radius of bulge is $20 \mu \mathrm{m}$ ( $1.5 \%$ of the droplet volume).

3) Defect in the Temperature Controller: Temperature is a critical factor in the PCR application, as the three steps in one cycle of PCR require distinct temperatures. Error in the temperature may degrade the performance of PCR, even cause the failure. For example, a wrong temperature during the annealing step can result in primers not binding to the template DNA at all. The error in temperature may result from the malfunction of the temperature controller. This defect affects fluidic parameters, i.e., viscosity of droplet $\mu_{d}$ and viscosity of filler fluid (silicone oil) $\mu_{0}$, but not geometrical parameters. In order to model the temperature error, the variation of viscosity versus temperature for droplet and filler fluid is analyzed [28], [29]. Here we assume linear interpolations in the temperature range $T$ of $25^{\circ} \mathrm{C} \sim 100{ }^{\circ} \mathrm{C}$ as follows:

- viscosity of silicone oil: $\mu_{0}=1.0955-0.0054 T(\mathrm{cP})$;

- viscosity of $0.1 \mathrm{M} \mathrm{KCL}: \mu_{d}=1.0225-0.0080 T$ (cP)

where test stimuli droplet is $0.1 \mathrm{M} \mathrm{KCL}$ and filler fluid is silicone oil. These linear interpolations are applied to (1), and we replace the fluidic parameters $\mu_{d}$ and $\mu_{0}$ with one temperature variable T

$$
\begin{aligned}
& \frac{\varepsilon_{0} \varepsilon_{R}}{2 d} V^{2}-F_{T}=B\left[\frac{U(1.0225-0.0080 T)}{\gamma_{L M}}\right]^{0.3} \gamma_{L M} \\
&+\left(\frac{\mathrm{mL}}{h}+s\right) U(1.0995-0.0054 T)+\zeta U
\end{aligned}
$$

Then, a similar approach is utilized to obtain the minimum detectable deviation for temperature in each step of PCR. Here we assume a 5\% tolerance for each physical parameter as well as the temperature. The experimental results show that the MDD of temperature is $13.8 \%$ for the denaturation step, $29.6 \%$ for the annealing step, and $19.4 \%$ for the synthesis step, making the denaturation step the most suitable phase for detecting defects in the temperature controller.

\section{CONCLUSION}

In this paper, we have presented a model for physical defects in droplet-based microelectrofluidic systems and a test development methodology for both operational and manufacturing defects. As microelectrofluidic systems become widespread in safety-critical biomedical applications, dependability emerges as a critical performance parameter. We have developed a cost- 
effective concurrent test methodology to increase the dependability of droplet-based microelectrofluidic systems. We have presented a classification of catastrophic and parametric faults in microelectrofluidic systems and shown how faults can be detected by electrostatically controlling and tracking droplet motion. A tolerance analysis method based on Monte-Carlo simulation has been developed to characterize the impact of parameter (both physical and fluidic) variations on system performance. We have also studies the use of droplet-based microelectrofluidic systems for real-time PCR and evaluated some typical defects in terms of their ease of detection. In our ongoing work, we are investigating the diagnosis of parametric faults that affect system performance.

\section{ACKNOWLEDGMENT}

The authors would like to thank Dr. T. Zhang of Cadence Design Systems and H. Ren of Duke University for their valuable comments. The work on droplet-based microelectrofluidic systems at Duke University has been pioneered by Prof. R. B. Fair, Dr. M. G. Pollack, and Dr. V. Pamula, and the authors would like to thank them for sharing their insights.

\section{REFERENCES}

[1] S. Venkatesh and Z. A. Memish, "Bioterrorism: a new challenge for public health," Int. J. Antimicrob. Agents, vol. 21, pp. 200-206, 2003.

[2] J. Paul, "Bioterrorism and biodefence," J. Infection, vol. 44, pp. 59-66, 2002.

[3] H. F. Hull, R. Danila, and K. Ehresmann, "Smallpox and bioterrorism: public-health responses," J. Lab. Clin. Med., vol. 142, pp. 221-228, 2003.

[4] M. G. Pollack, R. B. Fair, and A. D. Shenderov, "Electrowetting-based actuation of liquid droplets for microfluidic applications," Appl. Phys. Lett., vol. 77, pp. 1725-1726, 2000.

[5] T. Zhang, K. Chakrabarty, and R. B. Fair, Microelectrofluidic Systems: Modeling and Simulation. Boca Raton, FL: CRC, 2002.

[6] S. K. Cho, S. K. Fan, H. Moon, and C. J. Kim, "Toward digital microfluidic circuits: creating, transporting, cutting and merging liquid droplets by electrowetting-based actuation," in Proc. IEEE Conf. MEMS, Las Vegas, NV, Jan. 2002, pp. 32-52.

[7] V. Srinivasan, V. K. Pamula, M. G. Pollack, and R. B. Fair, "A digital microfluidic biosensor for multianalyte detection," in Proc. IEEE Conf. MEMS, Kyoto, Japan, Jan. 2003, pp. 327-330.

[8] M. G. Pollack, P. Y. Paik, A. D. Shenderov, V. K. Pamula, F. S. Dietrich, and R. B. Fair, "Investigation of electrowetting-based microfluidics for real-time PCR applications," in Proc. $\mu T A S$, Squaw Valley, CA, Oct. 2003, pp. 619-622.

[9] V. Srinivasan, V. K. Pamula, M. G. Pollack, and R. B. Fair, "Clinical diagnostics on human whole blood, plasma, serum, urine, saliva, sweat, and tears on a digital microfluidic platform," in Proc. $\mu$ TAS, Squaw Valley, CA, Oct. 2003, pp. 1287-1290.

[10] D. J. Holme and H. Peck, Analytical Biochemistry, 2nd ed. Essex, U.K.: Longman, 1993.

[11] International Technology Roadmap for Semiconductors (ITRS) [Online]. Available: http://public.itrs.net/Files/2003ITRS/Home2003.htm

[12] J. L. Jackel, S. Hackwood, J. J. Veslka, and G. Beni, "Electrowetting switch for multimode optical fibers," Appl. Opt., vol. 22, pp. 1765-1770, 1999.

[13] R. Yates, C. Williams, C. Shearwood, and P. Mellor, "A micromachined rotating yaw rate sensor," in Proc. Micromachined Devices and Components II, SPIE Meeting, Austin, TX, Oct. 1996, pp. 161-168.

[14] M. G. Pollack, "Electrowetting-based microactuation of droplets for digital microfluidics," Ph.D. dissertation, Dept. Elect. Comput. Eng., Duke Univ., Durham, NC, 2001.

[15] S. K. De and N. R Aluru, "Physical and reduced-order dynamic analysis of MEMS," in Proc. IEEE/ACM ICCAD, San Jose, CA, Nov. 2003, pp. 270-273.
[16] G. K. Fedder and Q. Jing, "A hierarchical circuit-level design methodology for microelectromechanical systems," IEEE Trans. Circuits Syst. II, Analog Digit. Signal Process., vol. 46, no. 9, pp. 1309-1315, Sep. 1999.

[17] A. Kolpekwar and R. D. Blanton, "Development of a MEMS testing methodology" (in Nov.), in Proc. ITC, Washington, DC, 1997, pp. 923-993.

[18] N. Deb and R. D. Blanton, "Analysis of failure sources in surface-micromachined MEMS," in Proc. ITC, Atlantic City, NJ, Oct. 2000, pp. 739-749.

[19] H. G. Kerkhoff, "Testing philosophy behind the micro analysis system," Proc. SPIE, vol. 3680, pp. 78-83, 1999.

[20] H. G. Kerkhoff and H. P. A. Hendriks, "Fault modeling and fault simulation in mixed micro-fluidic microelectronic systems," J. Electron. Test.: Theory Appl., vol. 17, pp. 427-437, 2001.

[21] H. G. Kerkhoff and M. Acar, "Testable design and testing of microelectro-fluidic arrays," in Proc. IEEE VTS, Napa Valley, CA, Apr. 2003, pp. 403-409.

[22] A. Jee and F. J. Ferguson, "Carafe: an inductive fault analysis tool for CMOS VLSI circuits," in Proc. IEEE VTS, Atlantic City, NJ, Apr. 1993, pp. 92-98.

[23] J. Ding, K. Chakrabarty, and R. B. Fair, "Scheduling of microfluidic operations for reconfigurable two-dimensional electrowetting arrays," IEEE Trans. Comput.-Aided Design Integr. Circuits Syst., vol. 20, pp. 1463-1468, Dec. 2001.

[24] H. Ren, R. B. Fair, M. G. Pollack, and E. J. Shaughnessy, "Dynamics of electro-wetting droplet transport," Sens. Actuators B, vol. 87, pp. 201-206, 2002.

[25] S. D. Nigam and J. U. Turner, "Review of statistical approaches to tolerance analysis," Comput.-Aided Design, vol. 27, pp. 6-25, 1995.

[26] J. R. Johnson, "Development of polymerase chain reaction-based assays for bacterial gene detection," J. Microbiol. Meth., vol. 41, pp. 201-209, 2000.

[27] T. Zhang, K. Chakrabarty, and R. B. Fair, "Behavioral modeling and performance evaluation of microelectrofluidics-based PCR systems using SystemC," IEEE Trans. Comput.-Aided Design Integr. Circuits Syst., vol. 23, pp. 843-858, 2004.

[28] CRC Handbook of Chemistry and Physics, 84th ed. Boca Raton, FL: CRC.

[29] Silicone Fluid Brochure From Gelest, Inc. [Online]. Available: http://www.gelest.com/Library/SiFluids.pdf

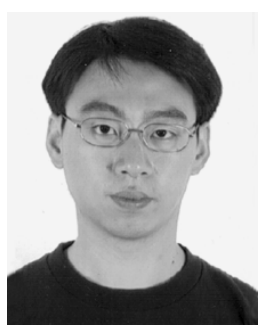

Fei Su (S'03) received the B.E. and the M.S. degrees in automation from Tsinghua University, Beijing, China, in 1999 and 2001, respectively, and the M.S. degree in electrical and computer engineering from Duke University, Durham, NC, in 2003. He is currently pursuing the $\mathrm{Ph} . \mathrm{D}$. degree in electrical and computer engineering at Duke University.

His research interests include the design and testing of mixed-technology microsystems, electronic design automation, mixed signal VLSI design, and MEMS modeling and simulation.

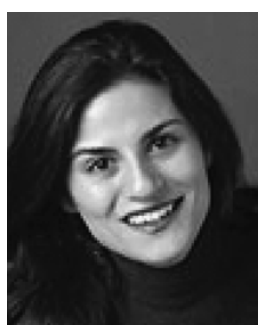

Sule Ozev (M'96) received the B.S. degree in electrical engineering from Bogazici University, Istanbul, Turkey, and the M.S. and Ph.D. degrees in computer science and engineering from the University of California at San Diego, La Jolla, in 1995, 1998, and 2002, respectively.

She is an Assistant Professor with the Electrical and Computer Engineering Department, Duke University, Durham, NC. Her research interests include system-level test methods for mixed-signal and RF circuits, computer-aided analysis of high-level and transistor-level circuits, and device modeling.

Dr. Ozev currently serves on the program committees of the International Test Synthesis Workshop (2004); the North Atlantic Test Workshop (2003 to 2004); the Symposium on Photonics, Networking, and Computing (2003 to 2004); the International Conference on CAD (2004); and the International Conference on Computer Design (2004). 


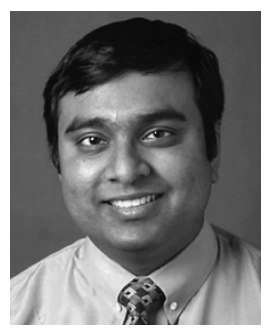

Krishnendu Chakrabarty (S'92-M'96-SM'00) received the B.Tech. degree in computer science and engineering from the Indian Institute of Technology, Kharagpur, in 1990, and the M.S.E. and Ph.D. degrees in computer science and engineering from the University of Michigan, Ann Arbor, in 1992 and 1995, respectively, all in computer science and engineering.

$\mathrm{He}$ is currently Associate Professor of electrical and computer engineering at Duke University, Durham, NC. He is a coauthor of two books: $\mathrm{Mi}$ croelectrofluidic Systems: Modeling and Simulation (Boca Raton, FL: CRC, 2002) and Test Resource Partitioning for System-on-a-Chip (Norwell, MA: Kluwer, 2002), and the Editor of SOC (System-on-a-Chip) Testing for Plug and Play Test Automation (Norwell, MA: Kluwer, 2002). He is also a coauthor of the forthcoming book Scalable Infrastructure forDistributed Sensor Networks (London, U.K.: Springer). He has published over 190 papers in journals and refereed conference proceedings, and he holds a U.S. patent in built-in self-test. His current research projects include the design and testing of system-on-chip integrated circuits; embedded real-time systems; distributed sensor networks; design automation of microfluidics-based biochips; and microfluidics-based chip cooling.

Dr. Chakrabarty is a member of ACM and ACM SIGDA, and a member of Sigma Xi. He is an Associate Editor of the IEEE TRANSACTIONS ON COMPUTER-AIDED DESIGN OF INTEGRATED CiRCUITS AND SYSTEMS and the IEEE TRANSACTIONS ON VERY LARGE SCALE INTEGRATION (VLSI) SySTEMS, and he served as an Associate Editor of the IEEE TRANSACTIONS ON CIRCUITS and Systems-PART II: ANAlog and Digital Signal Processing. He is an Associate Editor of ACM Journal on Emerging Technologies in Computing Systems and Editor of the Journal of Electronic Testing: Theory and Applications (JETTA). He serves as a subject area editor for the International Journal of Distributed Sensor Networks. He is a Distinguished Visitor of the IEEE Computer Society for 2005-2007. He serves as Vice Chair of Technical Activities in IEEE's Test Technology Technical Council, and is a member of the program committees of several IEEE/ACM conferences and workshops. He served as the Tutorial Co-Chair for the 2005 IEEE International Conference on VLSI Design and is the designated Program Co-Chair for the 2005 IEEE Asian Test Symposium. He is a recipient of the National Science Foundation Early Faculty (CAREER) award and the Office of Naval Research Young Investigator award. He is a recipient of a best paper award at the 2001 Design, Automation and Test in Europe (DATE) Conference. He is also a recipient of the Humboldt Research Fellowship from the Alexander von Humboldt Foundation, Germany. 